Short communication

\title{
Insights into (S)-rivastigmine inhibition of butyrylcholinesterase (BuChE): Molecular docking and saturation transfer difference NMR (STD-NMR)
}

\author{
Patrícia Bacalhau ${ }^{\mathrm{a}, \mathrm{b}, 1}$, Amor A. San Juan ${ }^{\mathrm{c}, 1}$, Albertino Goth ${ }^{\mathrm{c}}$, A. Teresa Caldeira ${ }^{\mathrm{a}, \mathrm{b}}$, Rosário Martins ${ }^{\mathrm{a}, \mathrm{b}}$, \\ Anthony J. Burke $\mathrm{a}^{\mathrm{a}, \mathrm{c}, *}$ \\ a Departamento de Química, Escola de Ciências e Tecnologia, Universidade de Évora, Rua Romão Ramalho, 59, 7000-671 Évora, Portugal

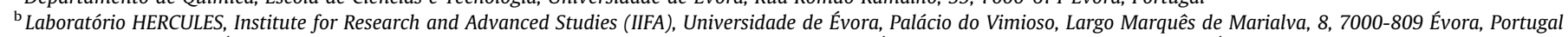 \\ ${ }^{\mathrm{C}}$ Centro de Química de Évora, Institute for Research and Advanced Studies (IIFA), Universidade de Évora, Rua Romão Ramalho, 59, 7000 Évora, Portugal
}

\section{A R T I C L E I N F O}

\section{Article history:}

Received 2 February 2016

Revised 7 May 2016

Accepted 2 June 2016

Available online 2 June 2016

\section{Keywords:}

Cholinesterase inhibition

Pseudo-irreversible inhibition

Rivastigmine

Molecular docking

STD-NMR

\begin{abstract}
A B S T R A C T
Rivastigmine is a very important drug prescribed for the treatment of Alzheimer's disease (AD) symptoms. It is a dual inhibitor, in that it inhibits both acetylcholinesterase (AChE) and butyrylcholinesterase (BuChE). For our screening program on the discovery of new rivastigmine analogue hits for human butyrylcholinesterase $(h \mathrm{BuChE})$ inhibition, we investigated the interaction of this inhibitor with BuChE using the complimentary approach of the biophysical method, saturation transfer difference (STD)NMR and molecular docking. This allowed us to obtain essential information on the key binding interactions between the inhibitor and the enzyme to be used for screening of hit compounds. The main conclusions obtained from this integrated study was that the most dominant interactions were (a) H-bonding between the carbamate carbonyl of the inhibitor and the $\mathrm{NH}$ group of the imidazole unit of $\mathrm{H} 434$, (b) stacking of the aromatic unit of the inhibitor and the W82 aromatic unit in the choline binding pocket via $\pi-\pi$ interactions and (c) possible $\mathrm{CH} / \pi$ interactions between the benzylic methyl group and the Nmethyl groups of the inhibitor and W82 of the enzyme.
\end{abstract}

(c) 2016 Elsevier Inc. All rights reserved.

\section{Introduction}

At the current time despite enormous advances in the field of medicine we are witnessing an exponential increase in the incidence of dementia in the global population. Estimates point to over 35.6 million people with dementia worldwide, a number that is expected to double every 20 years, and is expected to reach 115.4 million cases in 2050, 60-70\% of these cases have been assigned to Alzheimer's disease (AD) [1], the most common form of dementia with a progressive and irreversible neurodegeneration. AD is related to loss of cholinergic function, which affects memory, learning and behavior [2,3]. A large part of the strategies for treating $\mathrm{AD}$ have been based on the cholinergic hypothesis, which postulates that memory loss in Alzheimer's patients is associated with a deficit of cholinergic function in the brain [4]. The

\footnotetext{
* Corresponding author at: Departamento de Química, Escola de Ciências e Tecnologia, Universidade de Évora, Rua Romão Ramalho, 59, 7000-671 Évora, Portugal.

E-mail address: ajb@uevora.pt (A.J. Burke).

1 These authors contributed equally to this work.
}

loss of cholinergic neurons leads to the progressive reduction of acetylcholine $(\mathrm{ACh})$ in the brain and resulting cognitive impairment in $\mathrm{AD}$ [5]. ACh is hydrolyzed by both AChE and BuChE. In order to maintain AChE levels, in AD patients, acetylcholinesterase inhibitors (AChEIs) have been one of the prime drug targets for controlling $\mathrm{AD}$ at the symptomatic level $[6,7]$. Those currently prescribed include donepezil (Aricept), rivastigmine (Exelon), galantamine (Reminyl) and tacrine (Cognex) (Fig. 1) [8]. Tacrine is a reversible and a dual inhibitor of AChE and BuChE. It was approved in 1993 by the FDA. However, it has been withdrawn from the market, due to the hepatotoxicity of its metabolites [9]. As tacrine shows excellent binding for human AChE (hAChE) and human plasma BuChE ( $h p B u C h E$ ) with $\mathrm{IC}_{50}$ s of 190 and $47 \mathrm{nM}$, respectively, there is current interest in making tacrine analogues and conjugates $[10,11]$. Galantamine, a natural product, is a reversible and selective AChE inhibitor. It inhibits the degradation of ACh by binding in the AChE active site [8]. It demonstrated the following binding characteristics for heAChE and $h p B u C h E$ : $\mathrm{IC}_{50} \mathrm{~S}$ of 800 and $7300 \mathrm{nM}$, respectively [11]. Donepezil is a reversible and selective AChE inhibitor. It was approved by the FDA in 1996, and has given $\mathrm{IC}_{50} \mathrm{~S}$ of 22 and $4150 \mathrm{nM}$ for $h \mathrm{AChE}$ and $h \mathrm{BuChE}$ respectively 


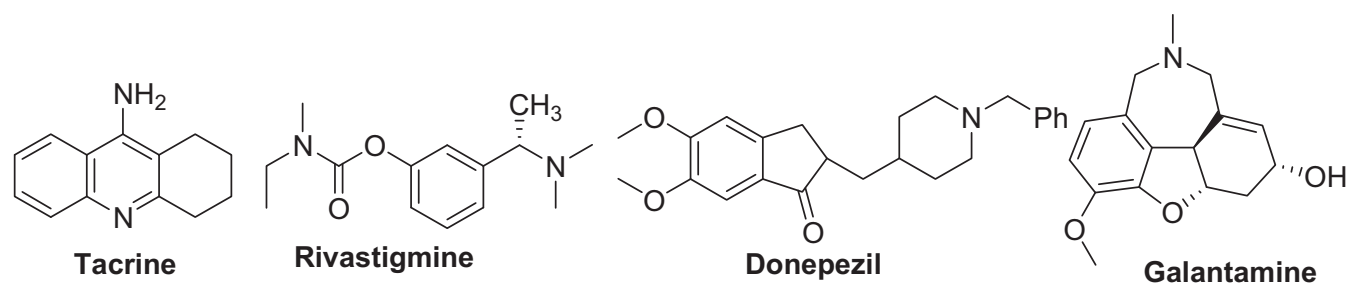

Fig. 1. Commercialized ChE inhibitors.

[11]. Rivastigmine is a pseudo-irreversible, dual inhibitor. It is known to carbamoylate a serine residue in the active site of AChE after binding. However, this is only transient and the enzyme reverts back to its original form upon hydrolysis of the enzymecarbamate bond [11]. It was shown to furnish $\mathrm{IC}_{50}$ values of 4150 and $37 \mathrm{nM}$ for the same ChEs as mentioned above [11]. Some of its key attributes are its facile blood-brain-barrier (BBB) permeability and it low degradation by the CYP450 cytochrome system [12].

Some important work has been published on the molecular nature of the interactions between donepezil [13] (see the work of Brus et al. below), galantamine [14] and tacrine [10,15] with AChE. These interactions have been studied either with X-ray crystallography or molecular modeling (docking). The molecular nature of AChE-rivastigmine conjugates has also been studied [16]. BuChE is an interesting target to study as its activity increases significantly during the latter stages of $\mathrm{AD}$. It has also been shown that increased BuChE activity also plays an important role in $A \beta-$ aggregation during the early stages of senile plaque formation [8b]. By contrast there is less information available on the molecular nature of the BuChE-rivastigmine interactions. Human BuChE ( $h \mathrm{BuChE}$ ) and human AChE ( $h \mathrm{AChE}$ ) share $65 \%$ amino acid sequence homology [17].

In the interest of developing more potent analogues of rivastigmine [18] for $h$ BuChE inhibition (which is currently a key goal of our group), and for refining our screening methods, we undertook a detailed study of the key interactions between BuChE and rivastigmine using both molecular modeling and saturation transfer difference (STD)-NMR. STD-NMR is a useful biophysical tool to map ligand epitopes in close contact with the protein and to determine the level of binding between the ligand and the protein [19$22]$. We have already successfully employed STD-NMR in the case of galantamine for determining the key interactions and binding affinities in some of our lead compounds [14c]. For this study we prepared an active site models based on $h \mathrm{BuChE}$ and $h \mathrm{AChE}$ which were tested with some isoquinolinone hit compounds [14c].

\section{Results and discussion}

\subsection{Molecular docking studies of BuChE-rivastigmine}

The X-ray crystal structure of $h \mathrm{BuChE}$ has already been solved, as well as an X-ray crystal structure of a $h \mathrm{BuChE}$-piperidinecontaining amide complex, besides this inhibitor gave an $\mathrm{IC}_{50}$ of $21.3 \mathrm{nM}$ and a dissociation constant of $2.7 \mathrm{nM}$ [23]. $h \mathrm{BuChE}$ is characterized by possessing the catalytic triad which is constituted by S198, E325 and H438, the hydrophobic residues L286 and V288, which define the acyl pocket, these changes make the binding with the bulkier butyrate substrate possible [23]. Residues F288 and F290 in AChE are replaced by L286 and V288 in BuChE [23]. The tryptophan residue W82, interacts with the choline moiety in the catalytic active site via a $\pi$-cation interaction. The residue D70 constitutes the peripheral anionic site (PAS). In order to gain new insights on the interaction of rivastigmine with $h \mathrm{BuChE}$, so that it could be applied in the screening of new rivastigmine analogues, we conducted some molecular docking with our model which we had developed in a previous study [14c]. For this computational method described elsewhere [14c] (see full details in the supplementary information section), we prepared the $h \mathrm{BuChE}$ active site from the $h \mathrm{BuChE}$ crystal structures obtained from the Protein data bank as input structure (see supplementary information). As we used EqBuChE for our bioassays and STD studies (see below), prior to conducting our molecular modeling studies we carried a sequence alignment study, between $E q B u C h E$ and $h$ BuChE (Fig. $S_{2}$, supplementary information). What we saw was an overall identity of $90 \%$ for BuChE, and no differences were detected for the amino acid sequences within the active sites between the human and non-human sources.

The enzymatic hydrolytic cleavage of acetylcholine (ACh) by either BuChE or AChE results in the formation of both choline and acetate (forming an acetylated serine moiety which is subsequently hydrolyzed to release the acetate) (Fig. 2). An X-ray crystallography study conducted by Bar-on et al. [24] revealed that the carbamyl moiety is covalently linked to the active-site serine (see discussion below and Figs. 2 and 3), with the leaving group (-)-S-3-[1-(dimethylamino)ethyl]phenol (NAP) being retained in the anionic site (see discussion below). Rivastigmine is a slow substrate of ChE and not a simple reversible inhibitor, it is characterized as a pseudo-irreversible inhibitor [24]. However, the decarbamylation process is usually slow for ChEs [24]. It should also be noted that NAP is also an inhibitor of ChE [24].

In the study by Bar-on et al. [24] which reported the crystal structure of an TcAChE-rivastigmine conjugate at $2.2 \AA$ resolution, they showed that rivastigmine was covalently linked to the active site S200 (equivalent to S198 in $h \mathrm{BuChE}$ ), with the leaving group, (-)-S-3-[1-(dimethylamino)ethyl]phenol (NAP), retained in the anionic site with close association to H440, F330 and W84 (Fig. 3). Since rivastigmine is a carboxyl-esterase the role of this serine unit which is part of the catalytic triad is crucial.

It was also observed that there was a significant movement of $\mathrm{H} 440$ (equivalent to $\mathrm{H} 438$ in $h \mathrm{BuChE}$ ) away from E327, from $2.52 \AA$ in the native form to $4.01 \AA$ in the TcAChE conjugate. This results in the disruption of the catalytic triad, which can explain the slow kinetics of reactivation [24]. In the TcAChE-rivastigmine conjugate, the amino group of the carbamate moiety, with its ethyl and methyl substituents, is oriented toward F288 in the acyl pocket $(3.2 \AA)$. There are also aromatic-aromatic interactions of NAP with W84 and F330 (Fig. 3). In the case of our docking studies, in order to simplify matters since we wished to develop a rapid in silico screening method, we maintained the intact structure within the enzyme active site and included two water molecules, since two water molecules were observed in the X-ray crystal structure of Bar-on et al. [24] (Fig. 4). Significant interactions were observed, the principle interactions being: (a) two H-bonds with the carbamate moiety of rivastigmine formed between the $\mathrm{NH}$ group of the imidazole unit of the $\mathrm{H} 438$ residue and the $\mathrm{C}=\mathrm{O}$ group ( $2.87 \AA$ ) (this trend was also observed in the study of Brus et al. [23]) and between the OH group of S198 and the carbonyl group (a trend again noted in the study of Brus et al. [23]) and (b) T120 


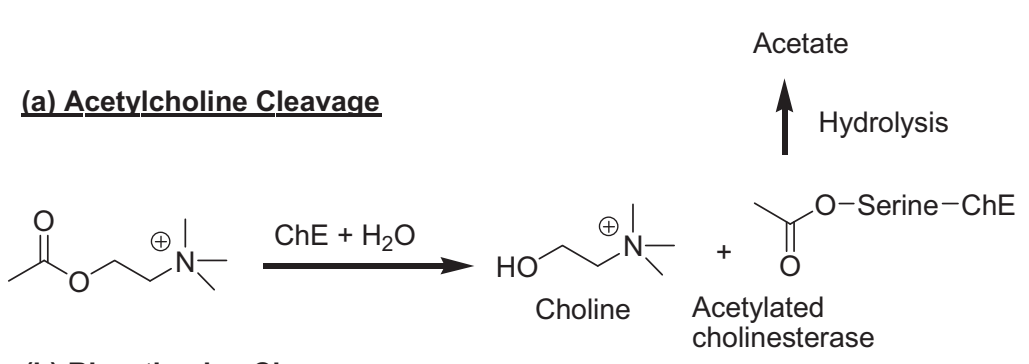

(b) Rivastigmine Cleavage

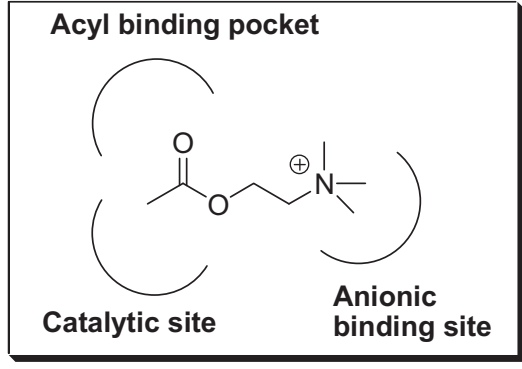<smiles>CCN(C)C(=O)Oc1cccc([C@H](C)N(C)C)c1</smiles>

Rivastigmine

(-)-S-3-[1-(dimethylamino) ethyl]phenol (NAP)
Carbamatylated cholinesterase

Fig. 2. Generalized scheme for cholinesterase catalyzed hydrolysis of (a) acetylcholine - including its mode of binding within the ChE active site and (b) rivastigmine.

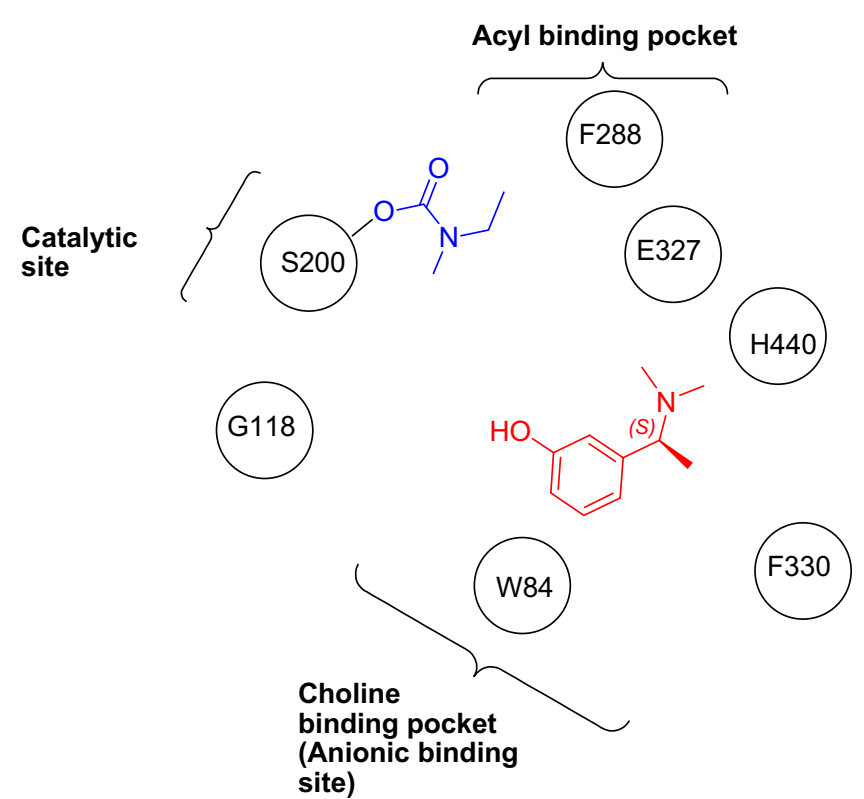

Fig. 3. Simple generalized depiction of the interaction of the cleaved rivastigmine moieties, (-)-S-3-[1-(dimethylamino)ethyl]phenol (NAP) (red) and carbamate (blue) with the TcAChE active site. Adapted from Ref. [24].

was observed to interact with one of the water molecules and both S79 and D70 with the other water molecule. There also appears to be a close interaction, which could possibly be a $\mathrm{CH} / \pi$ interaction [25] between W82 and the methyl groups of the NAP moiety - this of course is in line with the X-ray crystal analysis of Bar-on et al. [24] only in this case there is an intact inhibitor and the residue is W82 and not W84 (this type of interaction was also observed in the X-ray crystal structure of the $h \mathrm{BuChE}$-inhibitor conjugate of Brus et al. [23]) To validate this study we conducted the following STD-NMR study (see below).

\subsection{Saturation Transfer Difference - Nuclear Magnetic Resonance (STD - NMR)}

We performed a key STD-NMR experiment to map the ligandprotein interaction of rivastigmine (as its tartrate salt) with equine $E q \mathrm{BuChE}$ (this was chosen because of its commercial availability at

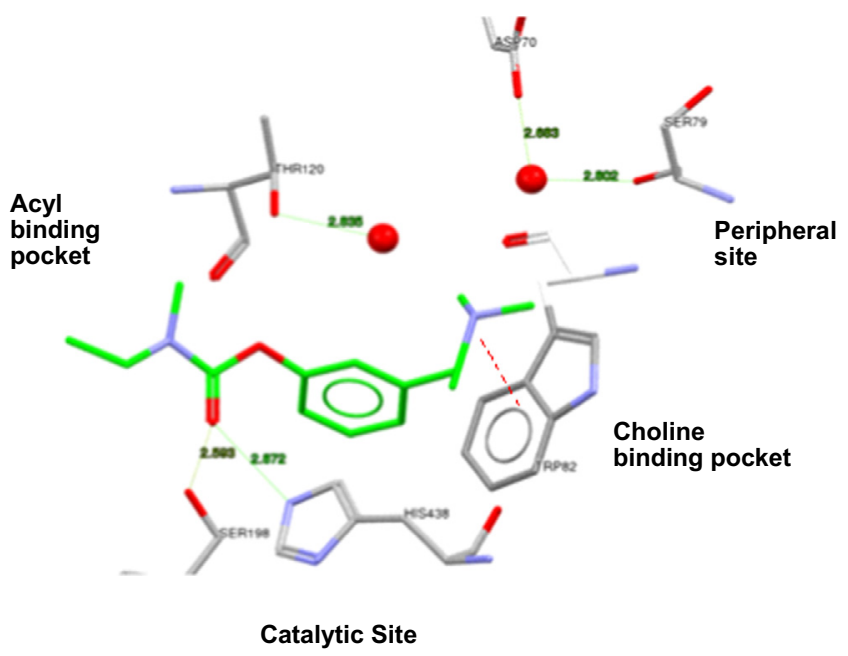

Fig. 4. The predicted binding mode of rivastigmine to the $h \mathrm{BuChE}$ active site. Hydrogen bonds are shown as green dotted lines, formed between the ligand rivastigmine (green color) with the residues in the active site (grey color). Water molecules in the active site are represented as red spheres. Note: the electrostatic non-covalent and the H-Bonding interactions are shown with green lines and, a possible $\mathrm{CH} / \pi$ interaction between W82 and the methyl and ethyl groups of the NAP moiety is shown with a broken red-line. The assignment of the binding regions is based on Ref. [17].

low price). To precisely map ligand epitopes in close contact with the protein, we acquired STD build up curves by obtaining spectra at different saturation times [19-22]. The observed STD amplification $\left(\mathrm{A}_{\mathrm{STD}}\right)$ is not the same for all the hydrogens in rivastigmine. This is due to the fact that not all the proton signals in the STDNMR spectrum received the same amount of saturation $[19,20]$. Thus, the distribution of saturation transferred among the different compound protons indicates spatial proximities between the protons of the compound molecule and the enzyme in the bound state [26]. A stronger intensity of a ligand's signal in the STD-NMR spectrum indicates closer inter-hydrogen distances between the ligand proton and the receptor surface in the bound state [20]. To quantitatively express the relative STD effects at a given saturation time, all of the STD signals are normalized against the most intense signal, which is arbitrarily assumed to be $100 \%$ [26]. It can be concluded that protons with relative STD values close to $100 \%$ belong to parts of the ligand that are very intimately recognized 
by the receptor binding-pocket, and hence must be regarded as significant for the interaction.

Galantamine was previously studied with EeAChE (commercially available and cheap) [14c]. We conducted an STD-NMR study of the complex formed between EeAChE and rivastigmine (Fig. 5). In the case of rivastigmine the aromatic group of the
NAP unit showed high STD enhancement in agreement with both X-ray crystallography and with our docking study which was most likely due to both $\pi-\pi$ and $\mathrm{CH} / \pi$ interactions with the tryptophan residue in the choline binding pocket and the hydrophobic residues in the PAS. In addition, both the benzylic methyl group (93\%) and the N-methyl groups (38\% each) of the

(a)

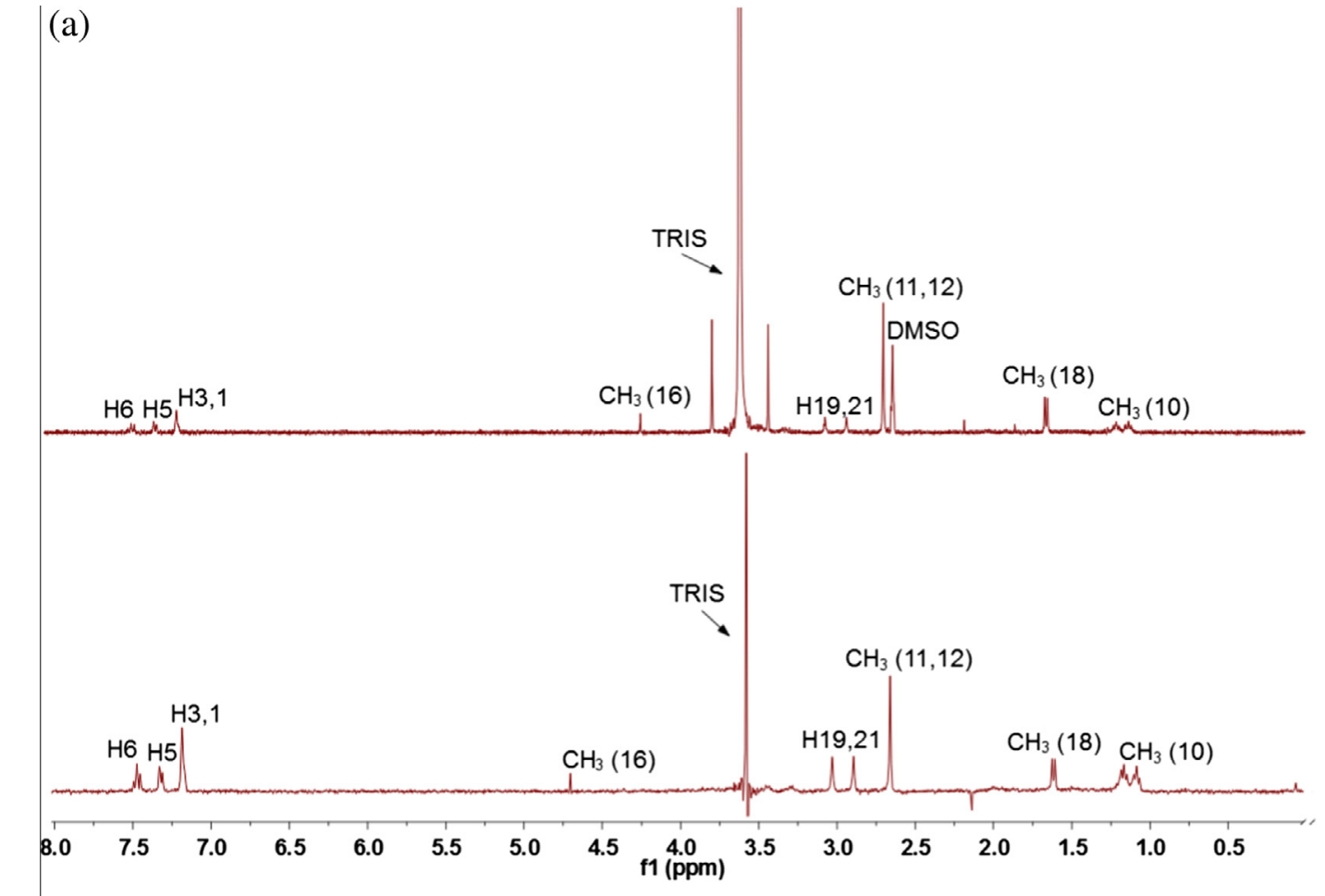

(b)

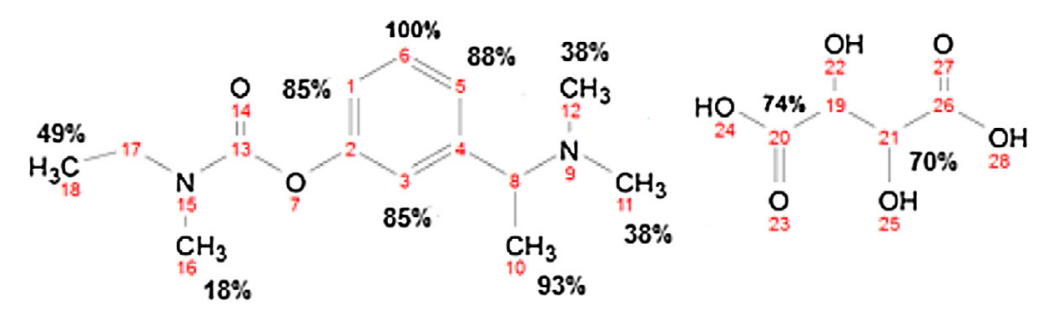

(c)

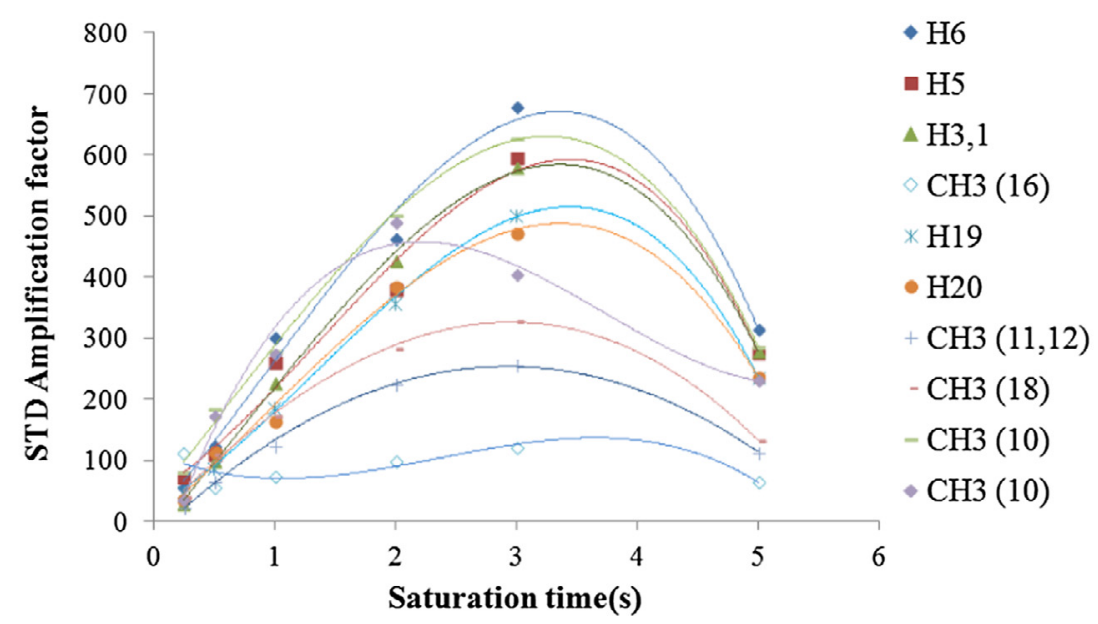

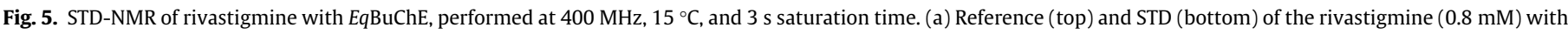

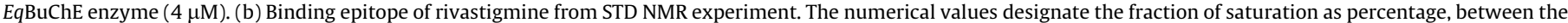

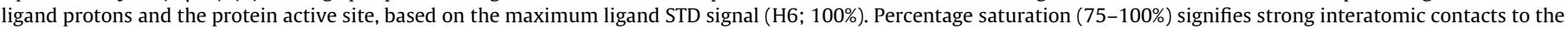
EqBuChE active site. (c) STD amplification factor as a function of saturation time for a 200-fold ligand excess. 
NAP unit showed significant enhancements supporting again $\mathrm{CH} / \pi$ interactions with hydrophobic residues in the PAS. The N-ethyl group of the carbamate unit showed an intensification of almost $49 \%$, most probably due to approximation to Y120.

As a final note, the tartrate moiety of the rivastigmine also showed some significant enhancement at the $\mathrm{H}-19$ and $\mathrm{H}-21$ (74\% and 68\%, respectively, Fig. 5). However, it is unknown whether this can interfere with the rivastigmine-enzyme binding.

\section{Conclusions}

When screening for hit and lead compounds, nimble yet reliable biophysical and/or non-biological methods, can be very useful, saving both time and expense. In our efforts at screening for new rivastigmine analogue hits showing good affinity for $h \mathrm{BuChE}$ we required rapid and reliable biophysical protocols to determine their binding affinities. We carried out a thorough molecular modeling docking study of rivastigmine in the active site of a $h \mathrm{BuChE}$ construct based on X-ray crystal structures and validated this by carrying out an STD-NMR study of the same inhibitor with $E q B u C h E$. The main conclusions that we obtained from these studies were that for the design of appropriate rivastigmine analogues the essential interactions appear to be (a) $\pi-\pi$ and $\mathrm{CH} / \pi$ interactions between the aromatic unit of the inhibitor with the W82 in the choline binding pocket and (b) the benzylic methyl group and the N-methyl groups of the NAP unit most probably with W82. Thus it seems important that potent rivastigmine analogues contain such pharmacophores in these positions relative to the enzyme active site residues. Currently we are preparing novel rivastigmine analogues based on this information, these results will be reported in due course.

\section{Acknowledgment}

The authors gratefully acknowledge the following funding sources: the INMOLFARM - Molecular Innovation and Drug Discovery (ALENT-57-2011-20) of the FEDER-INALENTEJO program ALENT-07-0224-FEDER-001743, and for grants to ASJ (post-doc), PB and AG. The Foundation for Science and Technology (FCT) in Portugal for funding through the strategic project PEst-OE/QUI/ UI0619/2014.

\section{Appendix A. Supplementary material}

Includes information on the, STD NMR and molecular modeling methods. Supplementary data associated with this article can be found, in the online version, at http://dx.doi.org/10.1016/j.bioorg. 2016.06.002.

\section{References}

[1] M. Burke, Chem. World 8 (August) (2014); R.J. Hargreaves, ACS Med. Chem. Lett. 3 (2012) 862.

[2] D. Price, Ann. Rev. Neurosci. 9 (1986) 489.

[3] P. Kasa, Z. Rakonczay, K. Gulya, Prog. Neurobiol. 52 (1997) 511.

[4] P. Davies, A.J. Maloney, Lancet 2 (1976) 1403.

[5] M. Garcia-Ayllon, D. Small, J. Avilla, J. Sáez-Valero, Front. Mol. Neurosci. 4 (2011) 1.

[6] K. Ingkaninan, P. Temkitthawon, K. Chuenchom, T. Yuyaem, W. Thongnoi, J. Ethnopharm. (2003) 261.

[7] K. Ingkaninan, C. de Best, H. Irth, R. van der Heijden, A. Hofte, B. Karabatak, U. Tjaden, J. van der Greef, R. Verpoorte, J. Chromatogr. A (2000) 61.

[8] For key reviews see: (a) S. Sakya, K. Karki, in: J-J. Li, D.S. Johnson (Eds.), Modern Drug Synthesis, John Wiley \& Sons Inc, Hoboken, New Jersey, 2010; (b) P. Anand, B. Singh, Arch. Pharm. Res. 36 (2013) 375.

[9] R.C. Petersen, R.G. Thomas, M. Grundman, D. Bennett, R. Doody, S. Ferris, D. Alasko, S. Jin, J. Kaye, A. Levey, E. Pfeiffer, M. Sano, C.H. Van Dyck, L.J. Thal, New Engl. J. Med. 352 (2005) 2379-2388.

[10] H. Jin, T. Nguyen, M.-L. Go, Med. Chem. 4 (2014) 688-696.

[11] Q-s. Yu, X. Zhu, H.W. Holloway, N.F. Whittaker, A. Brossi, N.H. Greig, J. Med. Chem. 45 (2002) 3684-3691.

[12] M. Mehta, A. Adem, M. Sabbagh, Int. J. Alzheim. Dis. (2012), http://dx.doi.org/ $10.1155 / 2012 / 728983$.

[13] G. Kryger, I. Silman, J.L. Sussman, Structure 7 (1999) 297.

[14] (a) H.M. Greenblatt, G. Kryger, T. Lewis, I. Silman, J.L. Sussman, FEBS Lett. 463 (1999) 321;

(b) H.M. Greenblatt, C. Guillou, D. Guenard, A. Argaman, S. Botti, B. Badet, C. Thal, I. Silman, J.L. Sussman, J. Am. Chem. Soc. 126 (2004) 15405-15408; (c) P. Bacalhau, A.A. San Juan, C.S. Marques, D. Peixoto, A. Goth, C. Guarda, M. Silva, S. Arantes, A.T. Caldeira, R. Martins, A.J. Burke, Biorg. Chem. 67 (2016) 18.

[15] F. Nachon, E. Carletti, C. Ronco, M. Trovaslet, Y. Nicolet, J. Ludovic, P.-Y. Renard, Biochem. J. 453 (2013) 393-399.

[16] (a) R.J. Polinsky, Clin. Therap. 20 (1998) 634-637;

(b) P. Bar-On, C.B. Millard, M. Harel, H. Dvil, A. Enz, J.L. Sussman, I. Silman, Biochemistry 41 (2002) 3555-3564.

[17] B. Brus, U. Košak, S. Turk, A. Pišlar, N. Coquelle, J. Kos, J. Stojan, J.-P. Colletier, S. Gobec, J. Med. Chem. 57 (2014) 8167-8179.

[18] A. Genoni, M. Benaglia, E. Mattiolo, S. Rossi, P.C. Barrulas, A.J. Burke, Tetrahedron Lett. 56 (2015) 5752-5756.

[19] A. Viegas, J. Manso, F. Nóbrega, E. Cabrita, J. Chem. Educ. 88 (2011) 990.

[20] J. Angulo, P.M. Nieto, Eur. Biophys. J. 40 (2011) 1357.

[21] B. Meyer, T. Peters, Angew. Chem. Int. Ed. 42 (2003) 864.

[22] A. Daranas, S. Khatib, R. Lysek, P. Vogel, J. Gavin, Chemistry Open 1 (2012) 13.

[23] B. Brus, U. Košak, S. Turk, A. Pišlar, N. Coquelle, J. Kos, J. Stojan, J.-P. Colletier, S. Gobec, J. Med. Chem. 57 (2014) 8167.

[24] P. Bar-On, C.B. Millard, M. Harel, H. Dvil, A. Enz, J.L. Sussman, I. Silman, Biochemistry 41 (2002) 3555.

[25] M. Nishio, M. Hirota, Y. Umezawa, The CH/P Interaction: Evidence, Nature and Consequences, Wiley-VCH, New York, 1998.

[26] M. Mayer, B. Meyer, Angew. Chem. Int. Ed. 38 (1999) 1784. 